\title{
The latest designing principle on microstructure and lattice- structure for high-energy-density antiferroelectric materials in fast \\ discharging applications
}

Yizheng Bao a,b, Xianlin Dong a,b,c and Genshui Wang ${ }^{* a, b, c}$

a. Key Laboratory of Inorganic Functional Materials and Devices, Shanghai Institute of Ceramics, Chinese Academy of Sciences, 588 Heshuo Road, Jiading District, Shanghai 201800, P. R. China.

b. Center of Materials Science and Optoelectronics Engineering, University of Chinese Academy of Sciences, 19 Yuquan Road, Shijinshan District, Beijing 100049, P. R. China

c. State Key Laboratory of High Performance Ceramics and Superfine Microstructure, Shanghai Institute of Ceramics, Chinese Academy of Sciences, 1295 Dingxi Road, Changning District, Shanghai 200050, P. R. China

\section{Corresponding Author}

Genshui Wang*

E-mail: genshuiwang@mail.sic.ac.cn 

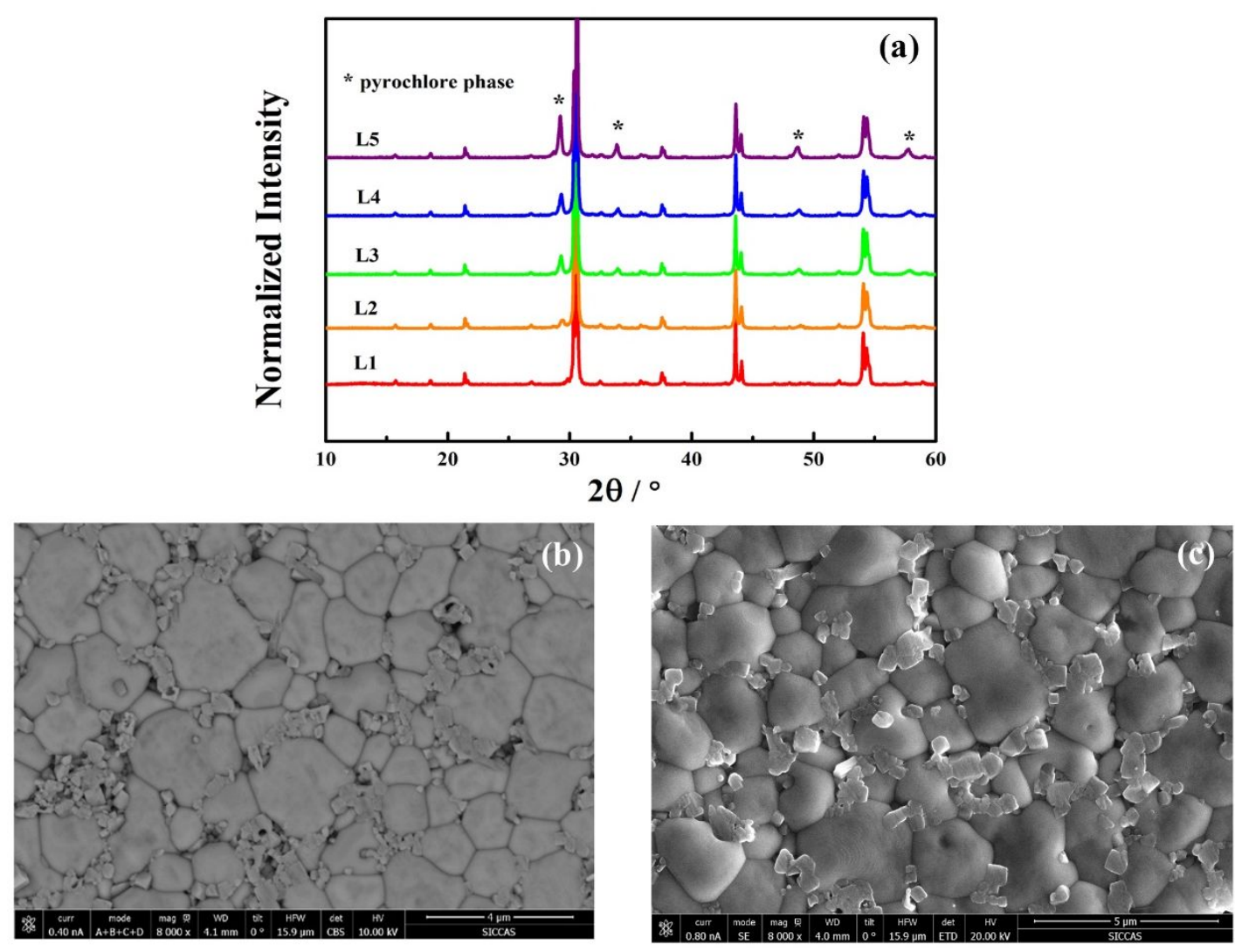

Fig. S1 Observation of the secondary phase born in the matrix of perovskite phase: a) XRD pattern of the five compositions b) SEM image (8000× magnification) on the mode of backscatter electrons c) on the mode of secondary electrons for the polished, thermally etched $\left(900^{\circ} \mathrm{C}, 0.5 \mathrm{~h}\right)$ surface for the composition $\mathrm{La}_{0.07} \mathrm{~Pb}_{0 \cdot 895}\left[\left(\mathrm{Yb}_{1 / 2} \mathrm{Nb}_{1 / 2}\right)_{0.92} \mathrm{Ti}_{0.08}\right] \mathrm{O}_{3}(\mathrm{~L} 4)$. 


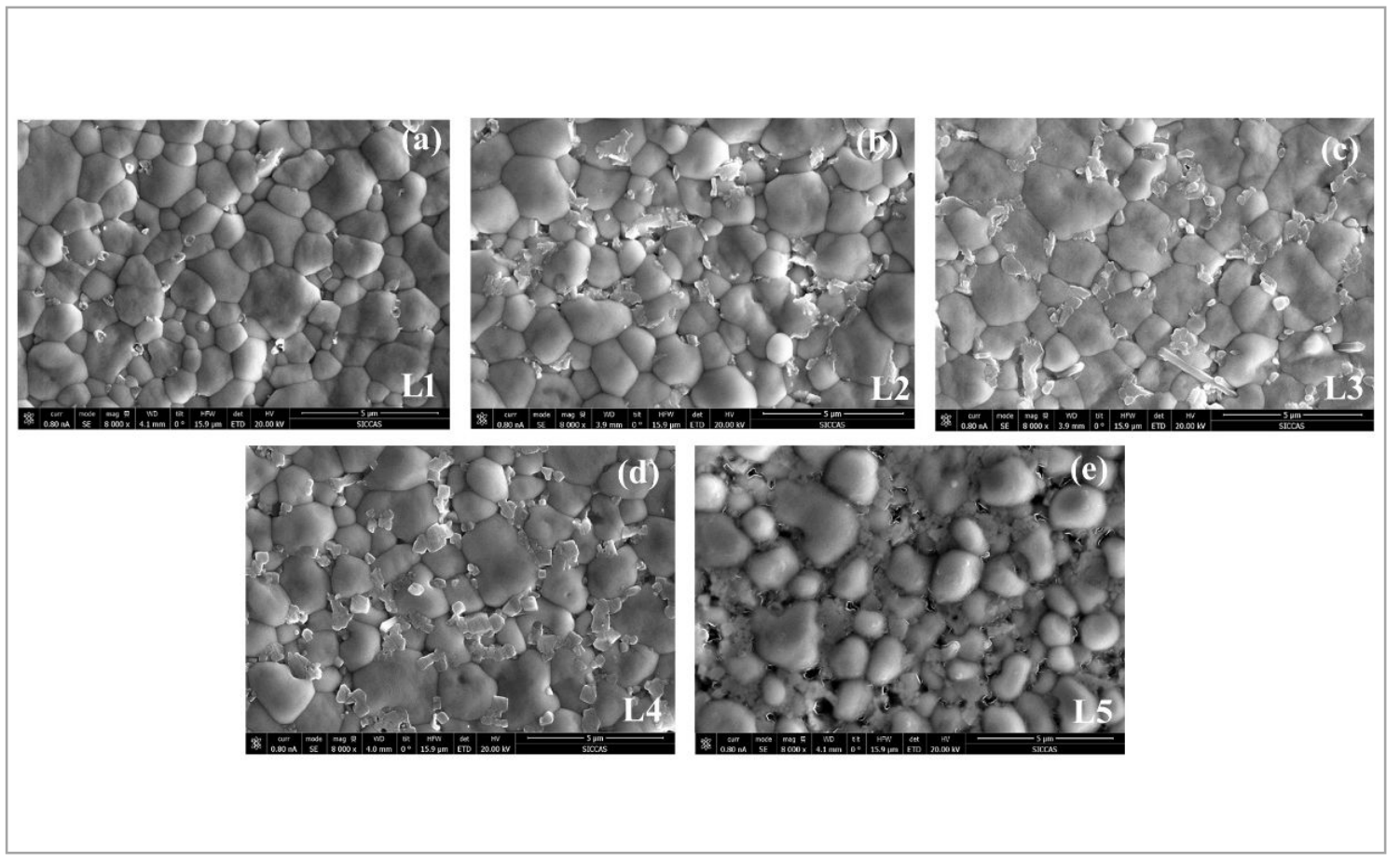

Fig. S2 SEM images (secondary electron mode, 8000× magnification) of the polished, thermally etched $\left(900^{\circ} \mathrm{C}, 0.5 \mathrm{~h}\right)$ cross-sections for L1, L2, L3, L4 and L5 

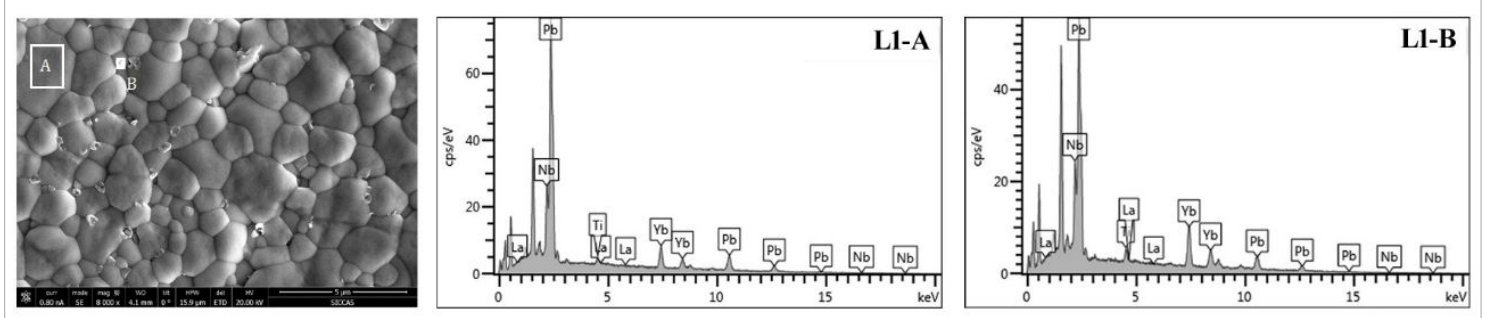

L1-A

\begin{tabular}{|c|c|c|c|c|c|c|}
\hline Element & $\begin{array}{l}\text { Line } \\
\text { Type }\end{array}$ & $\begin{array}{c}\text { Apparent } \\
\text { Concentration }\end{array}$ & k Ratio & $\mathrm{Wt} \%$ & $\begin{array}{l}\text { Wt\% } \\
\text { Sigma }\end{array}$ & Atomic \% \\
\hline $\mathrm{O}$ & & & & 12.66 & & 60.02 \\
\hline $\mathrm{Ti}$ & $\mathrm{K}$ series & 1.73 & 0.01730 & 1.24 & 0.13 & 1.97 \\
\hline $\mathrm{Nb}$ & L series & 13.83 & 0.13834 & 10.97 & 0.32 & 8.95 \\
\hline La & L series & 0.52 & 0.00462 & 0.36 & 0.29 & 0.20 \\
\hline $\mathrm{Yb}$ & L series & 30.13 & 0.30133 & 20.77 & 0.47 & 9.10 \\
\hline $\mathrm{Pb}$ & M series & 76.36 & 0.71062 & 53.99 & 0.54 & 19.76 \\
\hline Total: & & & & 100.00 & & 100.00 \\
\hline
\end{tabular}

L1-B
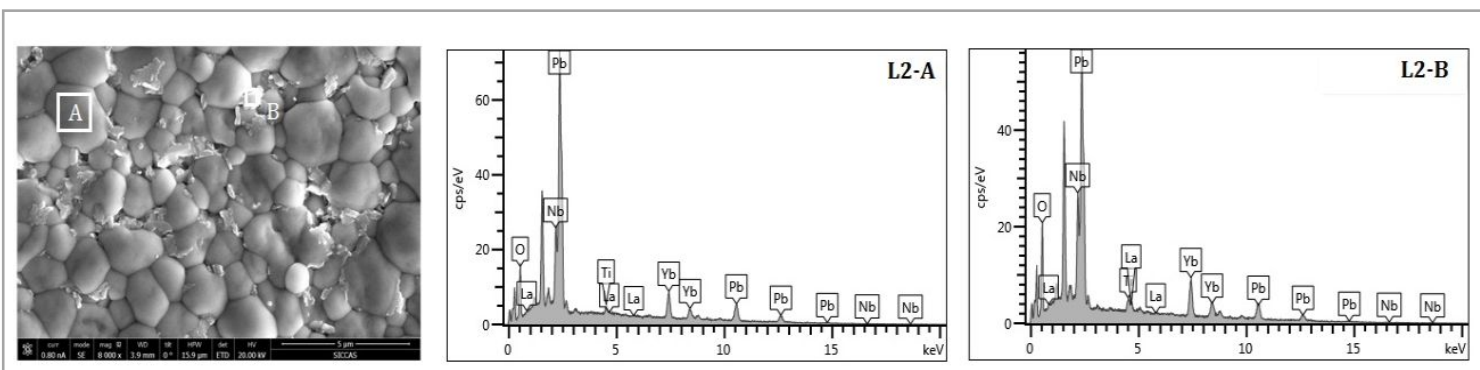

L2-A 


\begin{tabular}{|c|c|c|c|c|c|c|}
\hline Element & $\begin{array}{l}\text { Line } \\
\text { Type }\end{array}$ & $\begin{array}{c}\text { Apparent } \\
\text { Concentration }\end{array}$ & k Ratio & $\mathrm{Wt} \%$ & $\begin{array}{l}\text { Wt\% } \\
\text { Sigma }\end{array}$ & Atomic \% \\
\hline $\mathrm{O}$ & & & & 12.73 & & 60.09 \\
\hline $\mathrm{Ti}$ & $\mathrm{K}$ series & 1.81 & 0.01812 & 1.28 & 0.13 & 2.02 \\
\hline $\mathrm{Nb}$ & L series & 14.04 & 0.14045 & 10.98 & 0.33 & 8.93 \\
\hline La & L series & 0.96 & 0.00866 & 0.66 & 0.29 & 0.36 \\
\hline $\mathrm{Yb}$ & L series & 31.08 & 0.31081 & 21.08 & 0.46 & 9.20 \\
\hline $\mathrm{Pb}$ & M series & 76.43 & 0.71121 & 53.27 & 0.54 & 19.41 \\
\hline Total: & & & & 100.00 & & 100.00 \\
\hline
\end{tabular}

L2-B

\begin{tabular}{|c|c|c|c|c|c|c|}
\hline Element & $\begin{array}{l}\text { Line } \\
\text { Type }\end{array}$ & $\begin{array}{c}\text { Apparent } \\
\text { Concentration }\end{array}$ & k Ratio & $\mathrm{Wt} \%$ & $\begin{array}{l}\text { Wt\% } \\
\text { Sigma }\end{array}$ & Atomic \% \\
\hline O & & & & 13.87 & & 61.38 \\
\hline $\mathrm{Ti}$ & $\mathrm{K}$ series & 1.93 & 0.01931 & 1.41 & 0.13 & 2.08 \\
\hline $\mathrm{Nb}$ & $L$ series & 15.28 & 0.15282 & 12.99 & 0.35 & 9.89 \\
\hline La & L series & 3.72 & 0.03338 & 2.63 & 0.29 & 1.34 \\
\hline $\mathrm{Yb}$ & L series & 35.29 & 0.35287 & 25.23 & 0.48 & 10.32 \\
\hline $\mathrm{Pb}$ & $M$ series & 57.96 & 0.53934 & 43.87 & 0.53 & 14.99 \\
\hline Total: & & & & 100.00 & & 100.00 \\
\hline
\end{tabular}



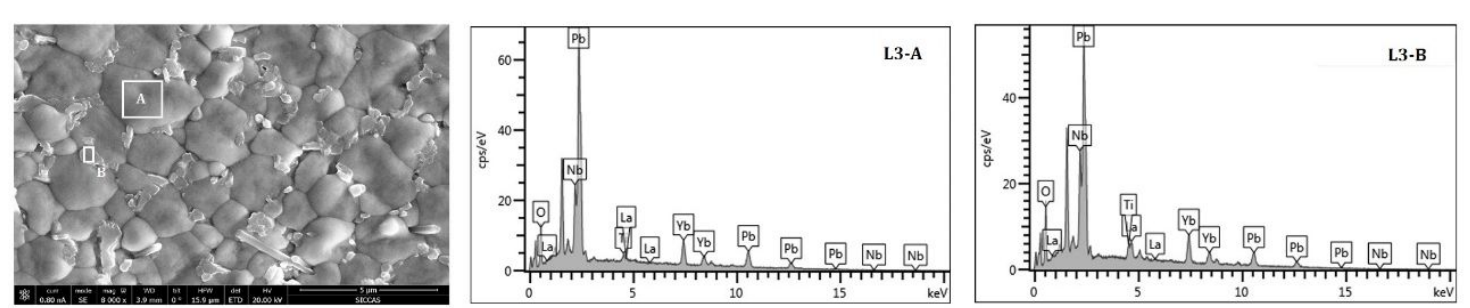

L3-A

\begin{tabular}{|c|c|c|c|c|c|c|}
\hline Element & $\begin{array}{l}\text { Line } \\
\text { Type }\end{array}$ & $\begin{array}{c}\text { Apparent } \\
\text { Concentration }\end{array}$ & k Ratio & $\mathrm{Wt} \%$ & $\begin{array}{l}\text { Wt\% } \\
\text { Sigma }\end{array}$ & Atomic \% \\
\hline $\mathrm{O}$ & & & & 12.56 & & 59.96 \\
\hline $\mathrm{Ti}$ & $\mathrm{K}$ series & 1.28 & 0.01276 & 0.94 & 0.13 & 1.50 \\
\hline $\mathrm{Nb}$ & $\mathrm{L}$ series & 13.05 & 0.13051 & 10.77 & 0.33 & 8.85 \\
\hline La & $\mathrm{L}$ series & 1.25 & 0.01117 & 0.89 & 0.29 & 0.49 \\
\hline $\mathrm{Yb}$ & $\mathrm{L}$ series & 31.44 & 0.31439 & 22.25 & 0.48 & 9.82 \\
\hline $\mathrm{Pb}$ & M series & 71.53 & 0.66561 & 52.58 & 0.55 & 19.38 \\
\hline Total: & & & & 100.00 & & 100.00 \\
\hline
\end{tabular}

\section{L3-B}

\begin{tabular}{|l|r|r|r|r|r|r|}
\hline \multicolumn{1}{|c|}{ Element } & \multicolumn{1}{|c|}{ Line } & \multicolumn{1}{|c|}{ Apparent } & k Ratio & \multicolumn{1}{|l|}{ Wt\% } & Wt\% & Atomic \% \\
\hline $\mathrm{O}$ & Type & Concentration & & \multicolumn{1}{c|}{ Sigma } & \\
\hline $\mathrm{Ti}$ & $\mathrm{K}$ series & 2.32 & 0.02317 & 1.67 & 0.14 & 2.41 \\
\hline $\mathrm{Nb}$ & L series & 16.02 & 0.16024 & 13.41 & 0.34 & 9.98 \\
\hline $\mathrm{La}$ & L series & 8.14 & 0.07305 & 5.71 & 0.31 & 2.84 \\
\hline
\end{tabular}




\begin{tabular}{|l|l|r|r|r|r|r|}
\hline $\mathrm{Yb}$ & L series & 31.27 & 0.31273 & 22.36 & 0.47 & 8.93 \\
\hline $\mathrm{Pb}$ & M series & 57.18 & 0.53210 & 42.58 & 0.52 & 14.21 \\
\hline Total: & & & & 100.00 & & 100.00 \\
\hline
\end{tabular}

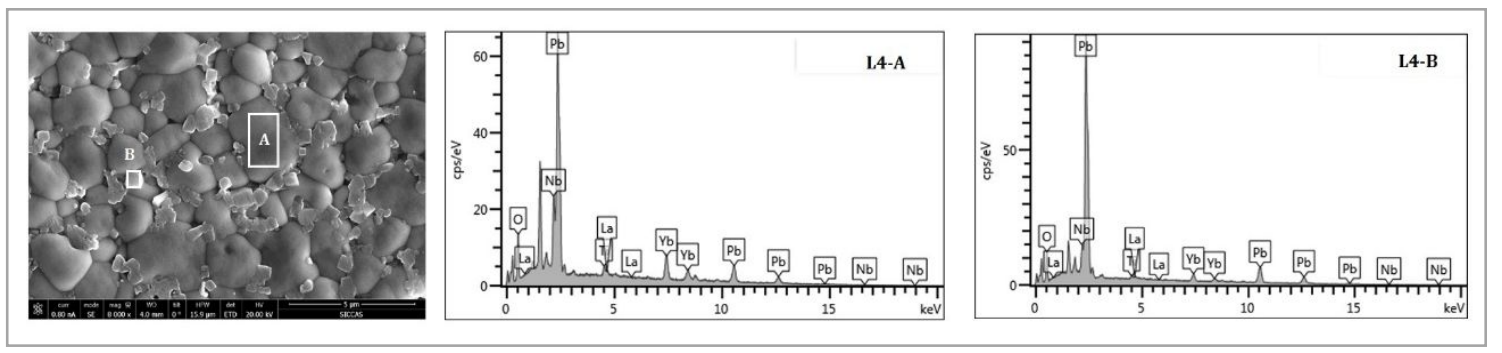

L4-A

\begin{tabular}{|c|c|c|c|c|c|c|}
\hline Element & $\begin{array}{l}\text { Line } \\
\text { Type }\end{array}$ & $\begin{array}{c}\text { Apparent } \\
\text { Concentration }\end{array}$ & k Ratio & $\mathrm{Wt} \%$ & $\begin{array}{l}\text { Wt\% } \\
\text { Sigma }\end{array}$ & Atomic \% \\
\hline 0 & & & & 12.91 & & 60.28 \\
\hline $\mathrm{Ti}$ & $\mathrm{K}$ series & 1.74 & 0.01741 & 1.28 & 0.13 & 1.99 \\
\hline $\mathrm{Nb}$ & $L$ series & 13.49 & 0.13489 & 11.12 & 0.32 & 8.94 \\
\hline La & L series & 2.37 & 0.02130 & 1.69 & 0.29 & 0.91 \\
\hline $\mathrm{Yb}$ & $L$ series & 30.69 & 0.30689 & 21.78 & 0.47 & 9.40 \\
\hline $\mathrm{Pb}$ & M series & 69.73 & 0.64888 & 51.22 & 0.54 & 18.47 \\
\hline Total: & & & & 100.00 & & 100.00 \\
\hline
\end{tabular}

L4-B

\begin{tabular}{|c|c|c|c|c|c|c|}
\hline Element & Line & Apparent & k Ratio & Wt\% & Wt\% & Atomic \% \\
& Type & Concentration & & & Sigma & \\
\hline
\end{tabular}




\begin{tabular}{|l|r|r|r|r|r|r|}
\hline $\mathrm{O}$ & & & & 14.42 & & 61.84 \\
\hline $\mathrm{Ti}$ & K series & 1.91 & 0.01907 & 1.41 & 0.13 & 2.02 \\
\hline $\mathrm{Nb}$ & L series & 15.34 & 0.15340 & 13.56 & 0.36 & 10.02 \\
\hline $\mathrm{La}$ & L series & 9.20 & 0.08252 & 6.61 & 0.32 & 3.27 \\
\hline $\mathrm{Yb}$ & L series & 34.20 & 0.34203 & 25.22 & 0.49 & 10.00 \\
\hline $\mathrm{Pb}$ & M series & 49.34 & 0.45912 & 38.79 & 0.53 & 12.85 \\
\hline Total: & & & & 100.00 & & 100.00 \\
\hline
\end{tabular}
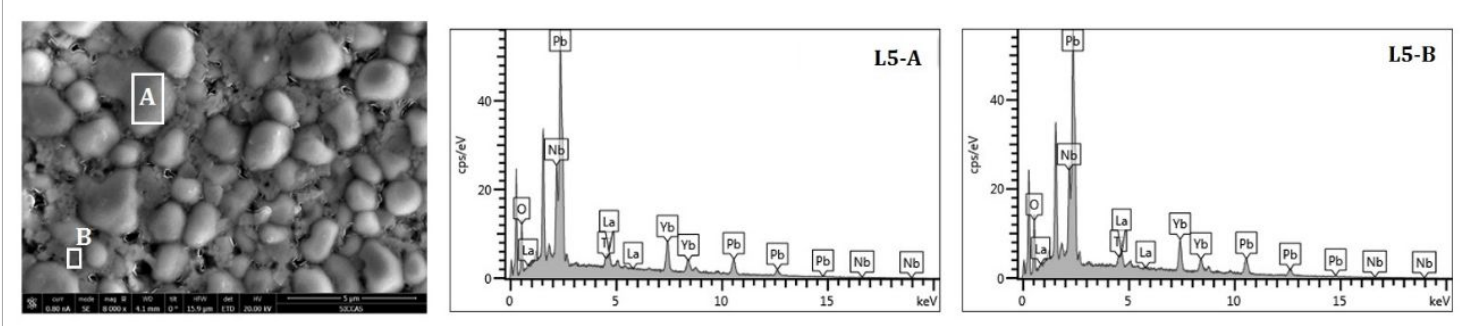

L5-A

\begin{tabular}{|c|c|c|c|c|c|c|}
\hline Element & $\begin{array}{l}\text { Line } \\
\text { Type }\end{array}$ & $\begin{array}{c}\text { Apparent } \\
\text { Concentration }\end{array}$ & k Ratio & $\mathrm{Wt} \%$ & $\begin{array}{l}\text { Wt\% } \\
\text { Sigma }\end{array}$ & Atomic \% \\
\hline $\mathrm{O}$ & & & & 12.70 & & 60.06 \\
\hline $\mathrm{Ti}$ & $\mathrm{K}$ series & 1.48 & 0.01483 & 1.08 & 0.13 & 1.71 \\
\hline $\mathrm{Nb}$ & $\mathrm{L}$ series & 13.35 & 0.13354 & 10.88 & 0.33 & 8.87 \\
\hline La & $L$ series & 2.49 & 0.02230 & 1.76 & 0.29 & 0.96 \\
\hline $\mathrm{Yb}$ & $\mathrm{L}$ series & 30.16 & 0.30160 & 21.21 & 0.46 & 9.28 \\
\hline $\mathrm{Pb}$ & M series & 72.13 & 0.67125 & 52.37 & 0.54 & 19.13 \\
\hline
\end{tabular}




\section{Total:}

100.00

100.00

L5-B

\begin{tabular}{|c|c|c|c|c|c|c|}
\hline Element & $\begin{array}{l}\text { Line } \\
\text { Type }\end{array}$ & $\begin{array}{c}\text { Apparent } \\
\text { Concentration }\end{array}$ & k Ratio & $\mathrm{Wt} \%$ & $\begin{array}{l}\text { Wt\% } \\
\text { Sigma }\end{array}$ & Atomic \% \\
\hline O & & & & 13.79 & & 61.23 \\
\hline $\mathrm{Ti}$ & $\mathrm{K}$ series & 1.77 & 0.01773 & 1.32 & 0.13 & 1.96 \\
\hline $\mathrm{Nb}$ & L series & 14.57 & 0.14567 & 12.63 & 0.34 & 9.66 \\
\hline La & L series & 6.61 & 0.05929 & 4.79 & 0.31 & 2.45 \\
\hline $\mathrm{Yb}$ & L series & 31.83 & 0.31827 & 23.37 & 0.49 & 9.59 \\
\hline $\mathrm{Pb}$ & M series & 57.15 & 0.53184 & 44.10 & 0.54 & 15.11 \\
\hline Total: & & & & 100.00 & & 100.00 \\
\hline
\end{tabular}

\begin{tabular}{|l|c|c|}
\hline & A: perovskite phase & B: pyrochlore phase \\
\hline L1 & $L a_{0.010} P b_{0.988}\left(Y b_{0.455} N b_{0.447} T i_{0.098}\right) O_{3}$ & $L a_{0.008} P b_{0.747}\left(Y b_{0.676} N b_{0.405} T i_{0.108}\right) O_{3}$ \\
\hline L2 & $L a_{0.018} P b_{0.969}\left(Y b_{0.459} N b_{0.446} T i_{0.101}\right) O_{3}$ & $L a_{0.066} P b_{0.733}\left(Y b_{0.504} N b_{0.483} T i_{0.102}\right) O_{3}$ \\
\hline L3 & $L a_{0.025} P b_{0.970}\left(Y b_{0.491} N b_{0.443} T i_{0.075}\right) O_{3}$ & $L a_{0.138} P b_{0.692}\left(Y b_{0.435} N b_{0.485} T i_{0.117}\right) O_{3}$ \\
\hline L4 & $L a_{0.045} P b_{0.920}\left(Y b_{0.468} N b_{0.445} T i_{0.099}\right) O_{3}$ & $L a_{0.168} P b_{0.635}\left(Y b_{0.440} N b_{0.474} T i_{0.124}\right) O_{3}$ \\
\hline L5 & $L a_{0.049} P b_{0.930}\left(Y b_{0.454} N b_{0.459} T i_{0.084}\right) O_{3}$ & $L a_{0.120} P b_{0.740}\left(Y b_{0.470} N b_{0.473} T i_{0.096}\right) O_{3}$ \\
\hline
\end{tabular}

Fig. S3 Raw EDS spectrums and output data for the marked area in L1, L2, L3, L4 and L5. For each composition, the perovskite phase was marked by A while pyrochlore phase was marked by B. The table below summarized all the normalized formula for the different 
phases in L1, L2, L3, L4 and L5.
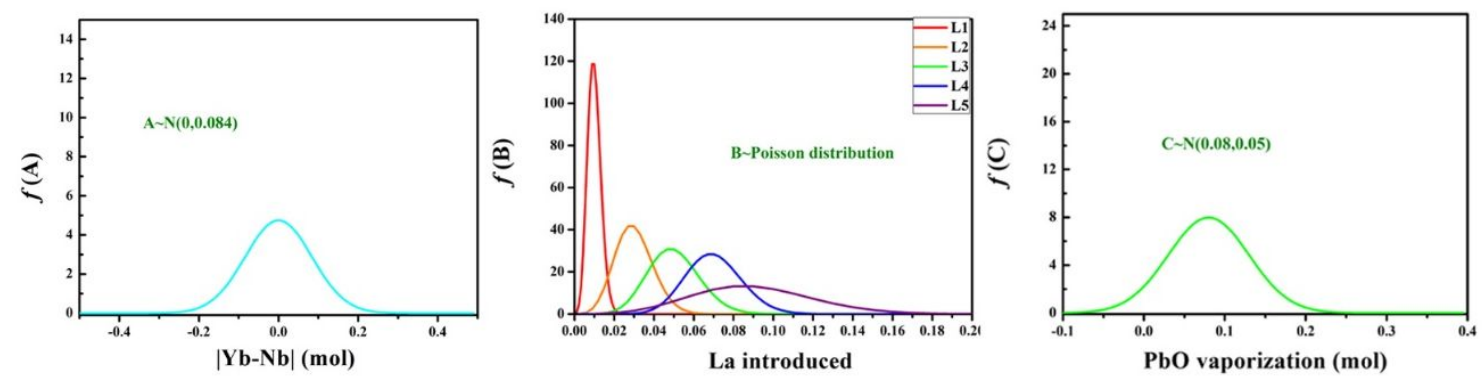

Fig. S4 The best fitted probability density distribution functions for three independent variables: A. concentration difference of $\mathrm{Yb} / \mathrm{Nb}(\mathrm{Yb}-\mathrm{Nb})$; B. introduced La concentration; C. vaporized $\mathrm{PbO}$ concentration.

$\mathrm{A}$ and $\mathrm{C}$ obey normal distribution, while $\mathrm{B}$ obeys Poisson distribution, and their density function distribution in line with EDS quantifying result in the Fig. S3. It is necessary to mention that all of the aforementioned distribution functions went through numbers of the revision in distribution parameters, back and forth, until the probability of forming pyrochlore phase $P(\mathrm{~A}, \mathrm{~B}, \mathrm{C})$ is reasonable. Fig. S4 show the best fitted distribution parameters and density distribution functions to secure the whole simulation in Fig. 3. 


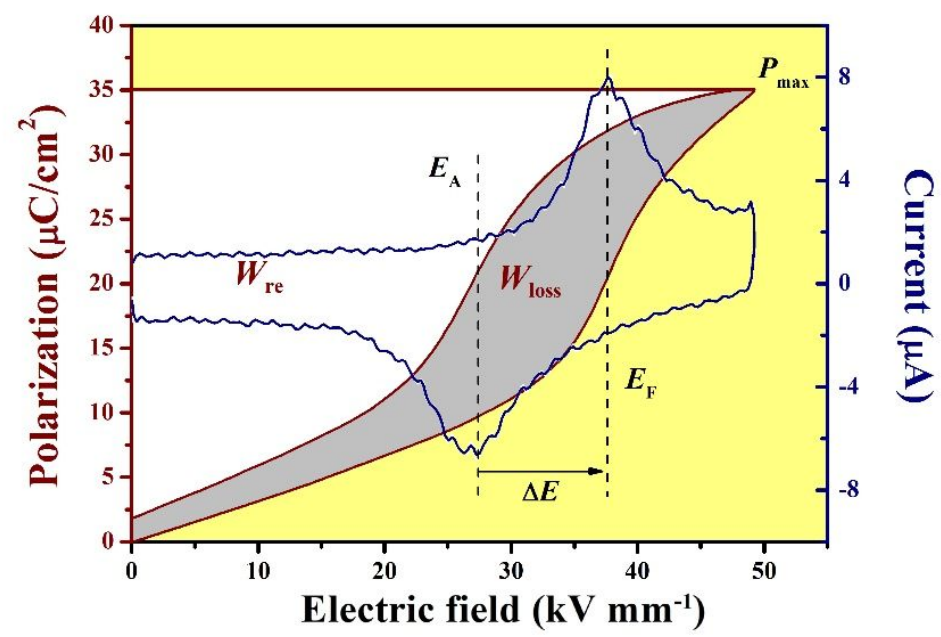

Fig. S5 Method to calculate energy storage properties and obtain energy storage parameters from the unipolar $P-E$ loop.

Charge Step: $W_{s t}=\int_{0}^{P_{\max }} E d P$

Discharge Step: $W_{r e}=-\int_{P_{\max }}^{P_{r}} E d P$

Energy loss: $W_{\text {loss }}=W_{s t}-W_{\text {re }}$

Energy Storage efficiency: $\eta=\frac{W_{r e}}{W_{s t}} \times 100 \%$

where $E, P, P_{\mathrm{r}}$ and $P_{\max }$ are external electric field, polarization, remnant polarization and maximum polarization, respectively.

Phase transition electric field $E_{\mathrm{F}}$ is obtained at positive current peak while $E_{\mathrm{A}}$ at negative current peak, naturally hysteresis loss is obtained by $\Delta E=E_{\mathrm{F}}-E_{\mathrm{A}} \cdot P_{\max }$ is found at maximum of polarization axis. 

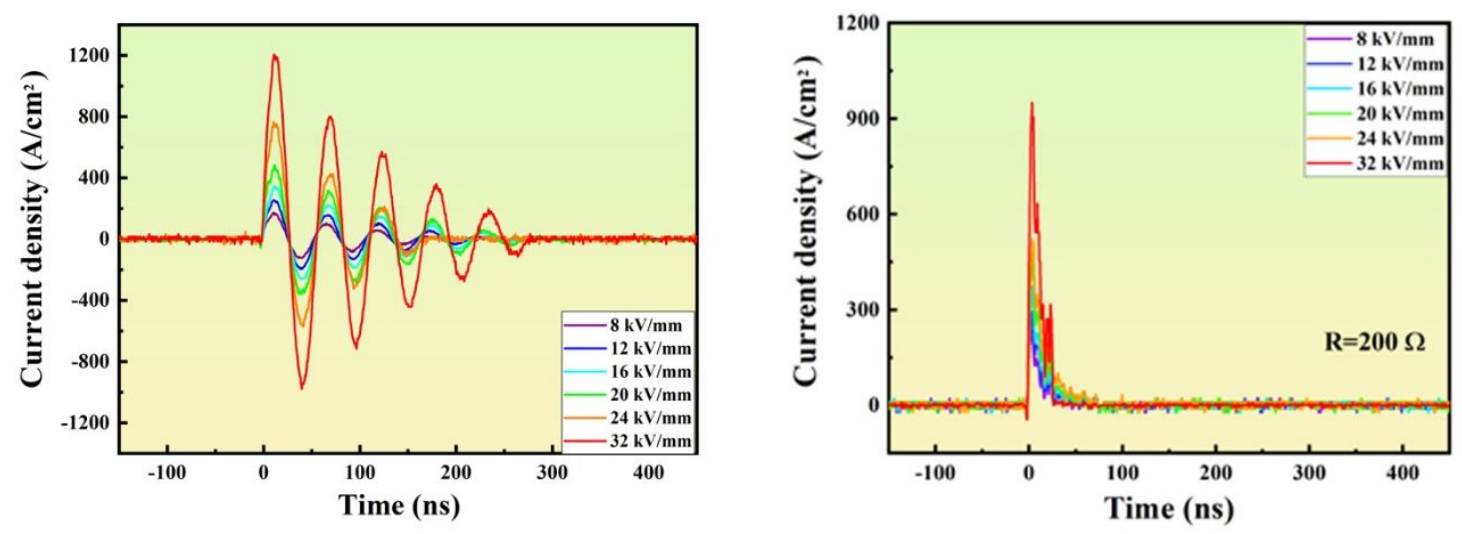

Fig. S6 Underdamped (left) and overdamped (right) discharging waveform of L4 under the increased electric field.
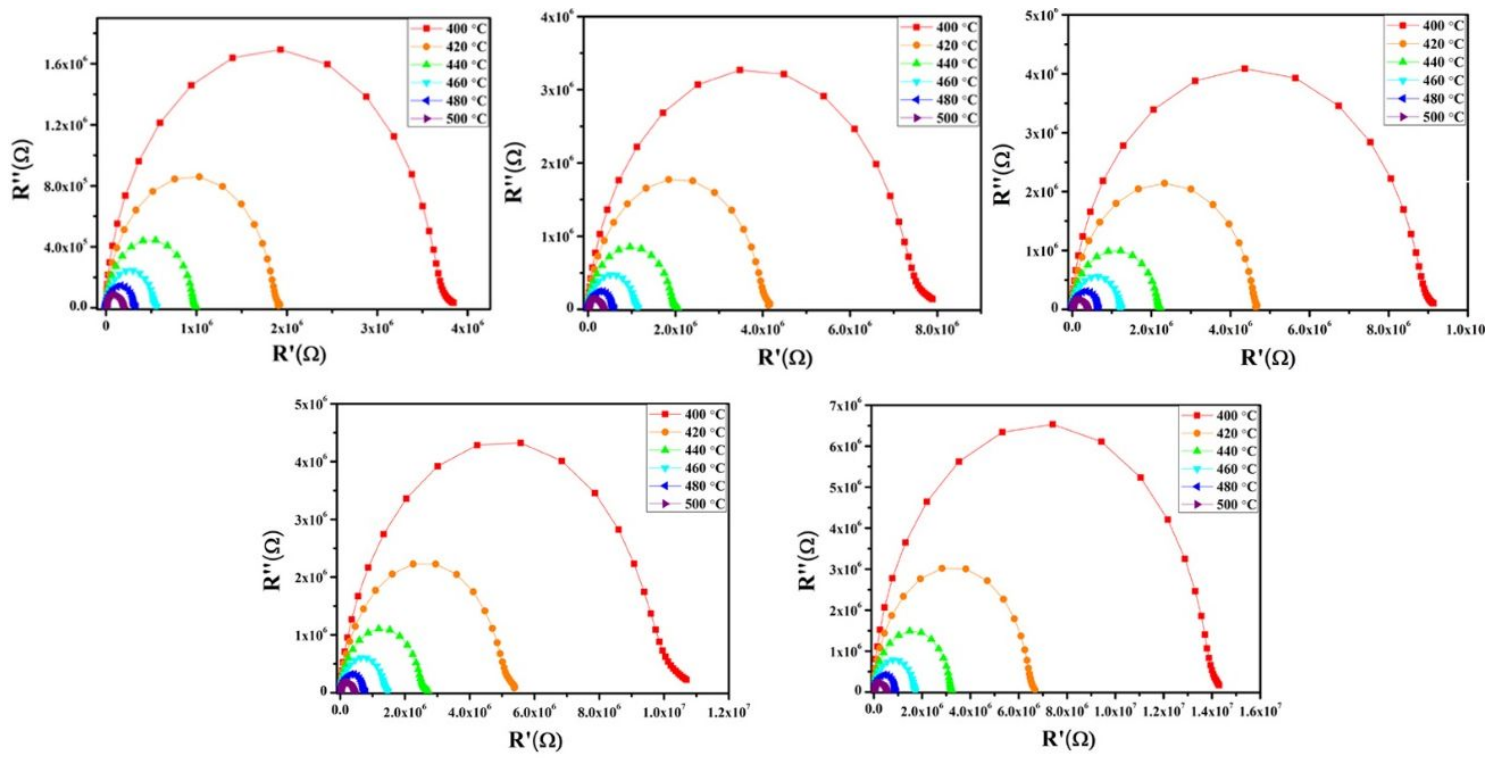

Fig. S7 Complex impedance spectrum of the five compositions (L1, L2 and L3 in the first row while L4 and L5 in the second from left to right) under the different temperatures: 400 ${ }^{\circ} \mathrm{C}, 420{ }^{\circ} \mathrm{C}, 440{ }^{\circ} \mathrm{C}, 460{ }^{\circ} \mathrm{C}, 480{ }^{\circ} \mathrm{C}$ and $500{ }^{\circ} \mathrm{C}$. 


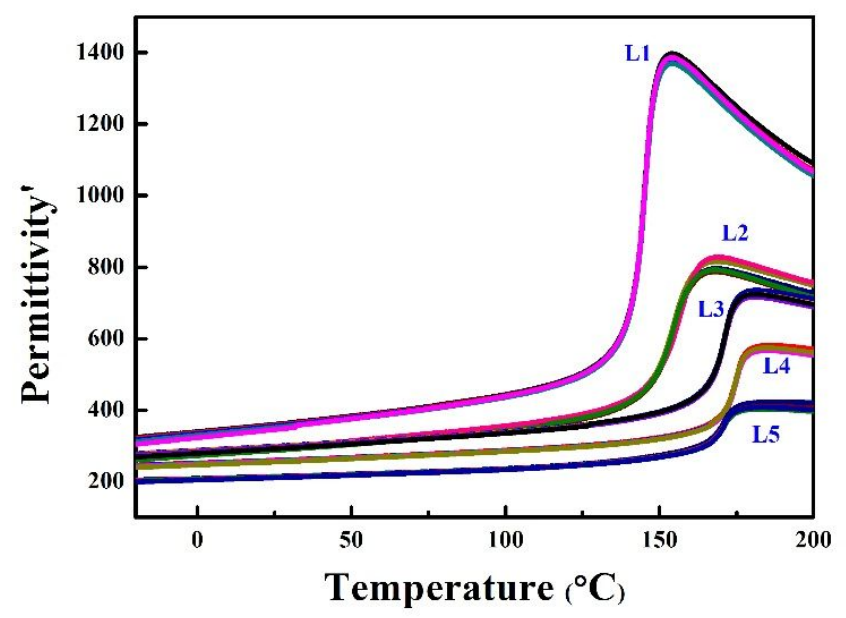

Fig. S8 Temperature dependence of permittivity for the five compositions

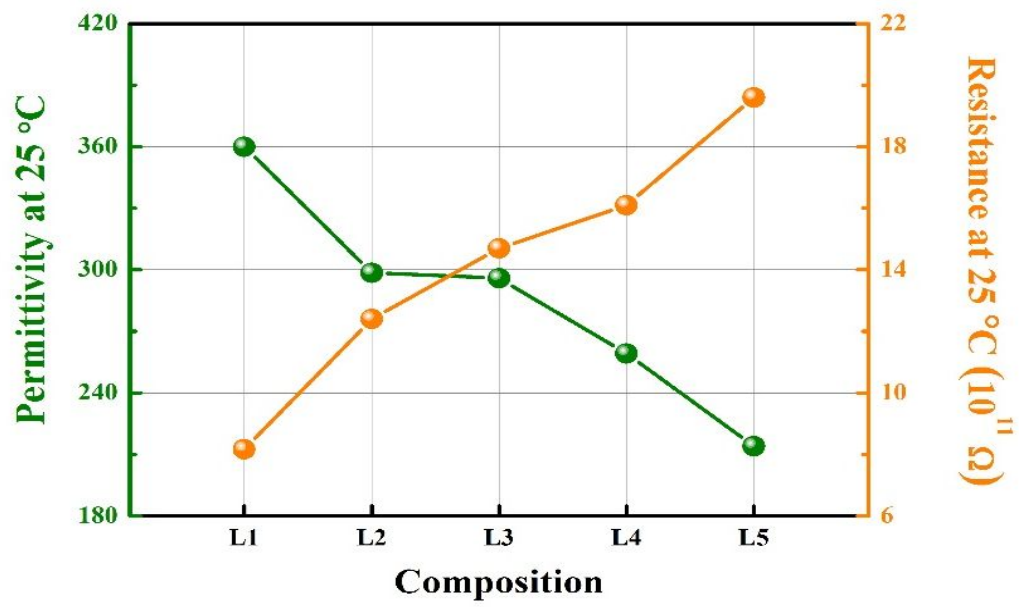

Fig. S9 Room temperature permittivity and resistance of the five compositions 


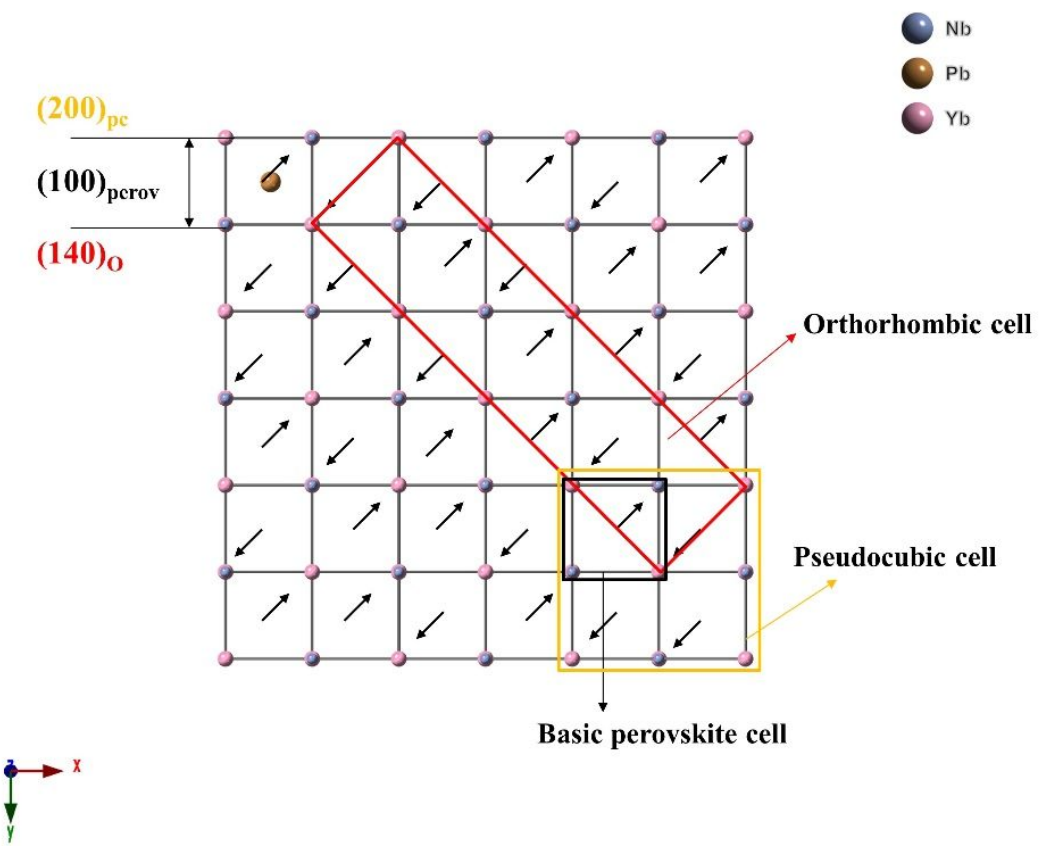

Fig. S10 Schematic diagrams of the projection of the lattice framework for PYN crystalline along $[001]_{\mathrm{pc}}$ axis and the different plane-indexing modes based on the different primitive cells.

\begin{tabular}{c|c|c|c|c}
\hline & $a(\AA)$ & $b(\AA)$ & $c(\AA)$ & $E(\mathrm{eV})$ \\
\hline NL- Pbnm & 17.80533 & 23.54833 & 8.2145 & -1677.2 \\
\hline L- Pbnm & 17.55972 & 23.29694 & 8.24381 & -1705.44 \\
\hline NL- P2 1 bn & 17.46962 & 23.42903 & 8.21442 & -1670.43 \\
\hline L- P2 1 bn & 17.45504 & 23.44305 & 8.2094 & -1692.38 \\
\hline
\end{tabular}

Table. S1 The lattice constant $(a, b$, and $c)$ and formation energy $E$ of the relaxed supercell for the four structures: NL-Pbnm, L-Pbnm, NL-P2 ${ }_{1}$ bn and L-P2 ${ }_{1} b n$. 